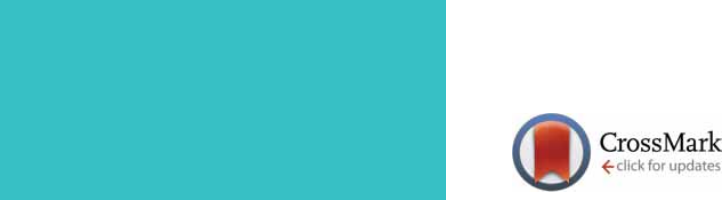

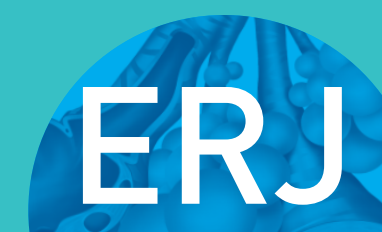

open research

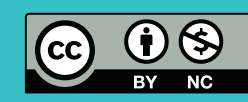

\section{Survey on accessibility and real-life application of noninvasive ventilation}

\author{
Szymon Skoczyński ${ }^{1}$, Raffaele Scala ${ }^{2}$ and Paolo Navalesi ${ }^{3}$
}

Affiliations: ${ }^{1}$ Dept of Pulmonology, School of Medicine in Katowice, The Medical University of Silesia, Katowice, Poland. ${ }^{2}$ Pulmonology and Respiratory Intensive Care Unit, S. Donato Hospital, Arezzo, Italy. ${ }^{3}$ Anesthesia and Intensive Care, Dept of Medical and Surgical Sciences, Magna Graecia University, Catanzaro, Italy.

Correspondence: Szymon Skoczyński, Dept of Pulmonology, School of Medicine in Katowice, The Medical University of Silesia, Ziołowa 45/47, 40-001 Katowice, Poland. E-mail: simon.mdsapoczta.fm

ABSTRACT Noninvasive mechanical ventilation (NIV) is an accepted method of respiratory failure treatment; however, at present, little is known about the global factors limiting NIV application.

A survey designed to determine NIV accessibility and limiting factors in world economies and regions was developed. The questionnaire was sent to members of the European Respiratory Society (ERS) Respiratory Intensive Care Assembly and all ERS National Delegates.

Replies to the survey were collected from 161 respondents from 46 countries. NIV was found to be provided most frequently by pulmonologists and intensivists. In high-income economies (HIEs), NIV reimbursement in chronic respiratory failure treatment was found to be independent of the underlying disease and supplementary insurance $(p<0.0001)$, whereas in upper-middle-income economies (UMIEs) it was found to be dependent on the underlying disease $(p<0.0001)$. In chronic respiratory failure, NIV was not reimbursed in lower-middle-income economies (LMIEs) $(p<0.0001)$. In LMIEs and UMIEs, the lack of financial resources was the main limiting factor in acute $(p=0.007)$ and chronic respiratory failure $(\mathrm{p}<0.0001)$. In the income-level-based assessment, financing was recognised as relevant in LMIEs and UMIEs $(p<0.0001)$, equipment in LMIEs and UMIEs $(p=0.03)$, medical staff in all economies $(p=0.02)$, and legal regulations in LMIEs $(\mathrm{p}=0.0005)$. It was confirmed that NIV in acute and chronic respiratory failure is reimbursed based on government regulations in UMIEs and HIEs $(p<0.0001)$, and is not reimbursed and probably will not be reimbursed in the near future in LMIEs $(p<0.0001)$.

We conclude that financial constraints are still considered a major limiting factor for NIV use.

@ERSpublications

NIV is still not included in university training but globally a lack of funds is the major factor limiting NIV use http://ow.ly/B3LL30lMPBu

Cite this article as: Skoczyński S, Scala R, Navalesi P. Survey on accessibility and real-life application of noninvasive ventilation. ERJ Open Res 2018; 4: 00062-2018 [https://doi.org/10.1183/ 23120541.00062-2018].

Received: May 252018 | Accepted after revision: Sept 072018

Copyright $\odot$ ERS 2018. This article is open access and distributed under the terms of the Creative Commons Attribution Non-Commercial Licence 4.0. 


\section{Introduction}

Noninvasive mechanical ventilation (NIV) is a rapidly emerging and increasingly cost-effective method used in the treatment of respiratory failure [1]. Unequal awareness, new treatment indications and rapid technical development may cause inequalities in NIV accessibility worldwide. Unfortunately, it has not yet been determined whether the reasons for these inequalities are country specific [2]. Surprisingly, in spite of "GOLD standard" NIV indications given in international guidelines, such as for acute exacerbations of chronic obstructive pulmonary disease (AE-COPD) [3], there is evidence that NIV is underused, even in well-developed Western countries [4]. This implies the need to identify reasons explaining the observed region- and/or income-related barriers impairing appropriate NIV application. We therefore designed a survey to assess the global accessibility of NIV, as well as to address the income- and/or region-specific conditions (i.e. financial and educational) that could correlate with inappropriate use of NIV in both acute and chronic respiratory failure.

\section{Material and methods \\ Survey}

The general aims of our survey were to: 1) assess discrepancies in NIV application among different geographical regions and countries grouped according to their income level in terms of NIV (i.e. supply, delivery, location of use, training and reimbursement), and 2) assess reasons for NIV underuse according to evidence level in acute and chronic respiratory failure.

To address the key questions, a survey on accessibility and real-life application of NIV was developed by the authors. The questionnaire was circulated for consultation with recognised experts in the field. A consensus was reached after three rounds of redistribution and discussion, and the final document was accepted.

The survey was aimed at members of the European Respiratory Society (ERS). As the ERS is a global scientific organisation that incorporates both physicians and allied health professionals, it was hoped that the assessed sample would provide responses from all groups of medical professionals. The survey was sent to the NIV Support Group in the ERS Respiratory Intensive Care Assembly. To ensure representative sample assessment, the survey was also sent to all ERS National Delegates using the SurveyMonkey (www. surveymonkey.com) online survey platform. The survey was opened in August 2014.

The responses obtained were grouped according to country region and country income based on World Bank definitions (low-income economies (LIEs), lower-middle-income economies (LMIEs), uppermiddle-income economies (UMIEs) and high-income economies (HIEs)) (tables 1 and 2) [5].

\section{Statistical analysis}

Survey results were summarised with the use of nonparametric statistics. To determine whether there were region- or income-related differences, we performed a univariate analysis using the Chi-squared test. Logistic regression analysis was performed to control for potential confounding effects of income and region. Statistical inference was based on $\mathrm{p}<0.05$.

\section{Results}

Response

Replies to the survey were collected from 161 respondents from 46 countries worldwide (tables 1 and 2), with a response rate of $42.5 \%$. However, there were no responses from LIEs. There were no relevant differences in sex or age distribution in the means of regional or national income.

\section{TABLE 1 Regional grouping}

Region description [5]

Asia, Australia and
Oceania
America
Africa
Europe

Armenia, Australia, China, India, Lebanon, New Zealand, Pakistan, Philippines, Saudi Arabia, Turkey 
TABLE 2 Income-related grouping

Income description (GNI per capita) [5]

Countries included

Low-income economies (SUSD1005)

Lower-middle-income economies

(USD1006-3955)

Upper-middle-income economies

(USD3956-12235)

High-income economies ( $\geqslant$ USD12236)
No responses

Armenia, Egypt, India, Pakistan, Philippines, Republic of Moldova, Tunisia

Argentina, Bosnia and Herzegovina, Brazil, Bulgaria, China, Croatia, Lebanon, Macedonia, Romania, Russia, Serbia, South Africa, Turkey

Australia, Austria, Belgium, Canada, Chile, Estonia, Finland, France, Germany, Greece, Hungary, Italy, Lithuania, Luxembourg, New Zealand, Norway, Poland, Portugal, Saudi Arabia, Slovakia, Slovenia, Spain, Switzerland, the Netherlands, UK, USA

GNI: gross national income.

\section{Staff members and training}

Medical doctors $(\mathrm{p}=0.009)$ were found to be the most frequently involved in NIV provision in all economies and regions (73.3\%), whereas nurses and respiratory therapists involved in NIV provision represented only $14.3 \%$ and $11.2 \%$, respectively. Only $20 \%$ of respondents were trained during their medical school education and this was not related to region or income.

\section{NIV access and constraints}

According to the univariate analysis, there were no region- or income-related differences concerning: medical specialisation most involved in NIV provision, setting where medical doctor training takes place, speciality of medical doctors who take part in postgraduate NIV training, reimbursement based on disease groups in acute respiratory failure, setting where NIV may be performed in acute respiratory failure, as well as in the way in which practical NIV training takes place (all $p>0.05$ ). There were also no differences in the age or sex of respondents (all $p>0.05$ ). All other responses that were found to reveal income- or region-related differences in NIV limitations are presented in table 3. The univariate analysis revealed that global differences in NIV accessibility and limiting factors are dependent on income rather than region (table 3).

Multivariate logistic regression analysis was performed to determine globally the independent NIV limitations after exclusion of possible confounding factors. The analysis revealed that financing in LMIEs and UMIEs is responsible for the major limitation in NIV implementation in acute $(\mathrm{p}=0.007)$ and chronic respiratory failure $(\mathrm{p}<0.0001)$.

In the assessment of risk factors responsible for limitation of home NIV in chronic respiratory failure, it was confirmed by logistic regression analysis that financing was relevant in regions 1 and 3 ( $p=0.02$ ). In the income-level-based assessment analysis, financing was recognised as important in LMIEs and UMIEs $(p<0.0001)$, equipment was recognised as an important factor in LMIEs and UMIEs $(p=0.03)$, medical understaffing was recognised to be relevant in all economies $(\mathrm{p}=0.02)$, and legal regulations were recognised to be relevant in LMIEs ( $\mathrm{p}=0.0005)$.

\section{NIV reimbursement}

There were no region- or income-related economic differences in the disease groups during the course of which NIV could have been reimbursed to treat acute respiratory failure patients. There were no significant differences in the influence of hospital setting on NIV reimbursement in acute respiratory failure.

The logistic regression analysis assessment confirmed that reimbursement for home NIV was independent of the underlying disease and of supplementary health insurance in HIEs $(\mathrm{p}<0.0001)$, and dependent on the underlying disease in UMIEs ( $\mathrm{p}<0.0001$ ). Domiciliary NIV was not provided in LMIEs, but patients could pay for the service/machine themselves $(\mathrm{p}<0.0001)$. Home NIV was found to be reimbursed if utilised for chronic respiratory failure during the course of COPD in UMIEs and HIEs $(p=0.009)$, neuromuscular diseases in UMIEs and HIEs ( $p=0.02$ ), obesity hypoventilation syndrome in UMIEs and HIEs ( $p=0.0004)$, chest wall deformities in UMIEs and HIEs $(p=0.004)$, and end-stage lung diseases for palliative care only in HIEs $(\mathrm{p}=0.02)$, but home NIV was not reimbursed when utilised in patients with interstitial lung diseases regardless of the national income level. 
TABLE 3 Univariate regional and financial survey analysis

Income-dependent differences

Question

Who is mostly involved in NIV practical
provision?

Is there a reimbursement when NIV is used to manage ARF?

NIV in ARF is reimbursed only if it is applied in: ...

\section{What are the reasons for the inappropriate use of NIV in ARF? \\ What are the factors that limit the use of NIV in ARF? \\ What are the determinants for reimbursement of home NIV in patients with CRF?}

What are the underlying diseases covered by the reimbursement for home NIV?
Region-dependent differences

Region-dependent differences
Difference
Medical doctors in regions 1,3
and 4
Only with private insurance in
region 4
Intensive care unit in regions 3
and 4
Respiratory ward in region 1
Setting in all regions
Financing in all regions
Independent of disease or
insurance in region 4
Dependent on disease in regions 1
and 3

COPD in all regions

0.04

Financing in regions 1 and 3

0.0001

Financing in regions 1-3

$<0.0001$ regions

0.03

0.04

0.04

0.02

0.05

0.05

$<0.0001$
Indications are based on

government regulations in all

0.03 p-value

$<0.0001$

What are the reasons for the inappropriate use of home NIV in a chronic setting?

What are the factors that limit home NIV in CRF?

At the moment NIV is reimbursed in both an acute and chronic setting in: ...

Difference p-value

Medical doctors in all economies

0.04

Not present

$>0.05$

Not present

$>0.05$

Financing in LMIEs and UMIEs

0.008

Financing in LMIEs and UMIEs

0.001

Independent of disease or insurance in HIEs

Dependent on disease in UMIEs

$<0.0001$

$<0.0001$

Not reimbursed; patients may pay in $\quad<0.0001$ LMIEs

COPD and chest wall deformities in UMIEs and HIEs

Neuromuscular diseases in UMIEs $\quad 0.009$ and HIEs

Obesity hypoventilation syndrome in $\quad 0.0002$ UMIEs and HIEs

End-stage lung disease palliative care $\quad 0.006$ in HIEs

Not reimbursed in all economies in $>0.05$

diffuse interstitial lung diseases Setting in all economies

Equipment in LMIEs and UMIEs Financing in LMIEs and UMIEs

Financing in LMIEs and UMIEs

Equipment in LMIEs and UMIEs Staff in all economies

Legal regulations in LMIEs

0.04

0.007

$<0.0001$

$<0.0001$

0.005

0.009

0.0007

All indications based on government regulations in UMIEs and HIEs

No and probably will be not reimbursed in the near future in

$<0.0001$

$<0.0001$

NIV: noninvasive ventilation; ARF: acute respiratory failure; CRF: chronic respiratory failure; COPD: chronic obstructive pulmonary disease; HIE: high-income economy; UMIE: upper-middle-income economy; LMIE: lower-middle-income economy. See tables 1 and 2 for region- and income-related groups.

It was confirmed that NIV in acute and chronic respiratory failure was reimbursed over a whole range of indications based on government regulations in UMIEs and HIEs $(\mathrm{p}<0.0001)$, and was neither reimbursed nor will be reimbursed in the near future in LMIEs $(\mathrm{p}<0.0001)$.

There were no regional or economic burdens restraining NIV implementation in hospital departments, regardless of whether the NIV introduced to hospital treatment was additionally reimbursed.

\section{Discussion}

The major strength of our study is its global approach. To the best of our knowledge, this was the first survey sent to a significant number of representatives from the various continents representing different levels of economies. The results have shown that financing is recognised worldwide as the most important factor responsible for NIV limitation in both acute and chronic respiratory failure in all economies, but the economic burden is highest in poorer countries (tables 2 and 3) [5]. Our findings are not in agreement with previously published work indicating that lack of physician knowledge, insufficient respiratory therapist 
training and inadequate equipment are the most important factors limiting NIV use [6, 7]. We have confirmed that medical doctors are still mainly involved in NIV provision, although respiratory therapists are well trained and may be cost-effective in comparison with employing medical doctors at the patient's bedside [8]. Unfortunately, respiratory therapists are available only in a minority of countries. For example, in Poland, chronic respiratory failure medical doctors are obliged to routinely visit NIV patients at home, whereas there is a dedicated nurse in high-dependency NIV rooms in hospitals (although the nurse is responsible only for the circuit and mask adjustments, not for settings adjustments or treatment initiation).

Although the aim of our survey was to concentrate on systemic burdens of performed NIV similarly to CRIMI et al. [9], we observed that NIV is being performed mostly by pulmonologists and anaesthesiologists, and to a much lesser extent by other specialists. We confirmed that in wealthy countries NIV reimbursement is based mainly on government regulations, which are not necessarily based on current medical guidelines or cost-effectiveness [1]. Unfortunately, chronic NIV use in LMIEs is neither reimbursed nor will be reimbursed in the near future, which indicates an urgent need for medical advocacy. There are also no major regional factors limiting NIV chronic respiratory failure application, with the exception of Africa and Asia.

In addition to the variability usually observed in general practice, there might be even larger differences between declared utilisation and real data concerning the numbers of patients treated. A study by MAHESHWARI et al. [7] found that, compared with guideline indications, in acute care specialised centres there was a tendency for NIV underuse in real-life situations of acute respiratory failure and acute-on-chronic respiratory failure in the course of AE-COPD and congestive heart failure. This might be even more pronounced in centres without NIV protocols. Another US-based survey found that the most important barriers to practical implementation of NIV in emergency departments were knowledge, availability of randomised controlled data and the time additionally required for NIV implementation [10]. This is partially confirmed by our findings, in which $<20 \%$ of respondents indicated having completed their NIV training at the level of a university course. This should be specifically addressed, because NIV training at a university level should be immediately implemented in all regions and economies. In contrast to these findings [10], we found no global (region- or income-related) differences resulting from equipment availability (although we have not assessed department-related local constraints in our survey). This can be explained by the fact that our respondents were mainly pulmonologists and anaesthesiologists who are expected to have more knowledge and practice in NIV application [11]. It should be emphasised that even in Canada and Europe, where NIV treatment is popular, unjustified underuse of NIV has been reported [12-14]. In contrast to the previous survey prepared by the ERS Respiratory Intensive Care Assembly officers [15], we obtained more responses in our survey from non-European countries and therefore we were able to mirror NIV application not only in Europe, but worldwide. In that previous survey, it was found that NIV was provided by different medical specialists [15], whereas our findings show that NIV is provided most frequently by medical doctors, without any income- or region-related differences in medical speciality. Identifying factors that limit NIV application is of high importance, especially because NIV may prolong life and improve the patient's quality of life in many common diseases or clinical situations $[1,9,16]$. In general, the fast increase in NIV use and improving success rates are nowadays secondary to a better choice of candidates and timing $[17,18]$. Unfortunately in COPD, even in European countries, NIV is frequently not implemented in clinical practice and there is a general underuse of NIV, including in situations such as AE-COPD [19]. This was mirrored by our findings, according to which AE-COPD was not considered as an independent indication for NIV reimbursement. Our survey was concentrated on global systemic aspects, and therefore we were not able determine whether NIV was used according to guidelines in terms of timing and clinical indications. Thus, in centres which confirmed implementing NIV, we are not able to verify that that method is being used in an inefficient way, delayed or in missed indications [20].

One of the most important and remarkable results of our survey was that financial constraints present in LMIEs have and will have a severely negative impact on NIV development. In those countries NIV is not reimbursed and probably will not be reimbursed in the near future, and therefore there is a high probability that in many centres NIV will not be used or will be provided in an unattended setting. On top of this, we need to emphasise that we did not obtain any responses from LIE countries, suggesting that NIV might not be used in those countries. This situation predisposes to underuse and/or application of low-standard, unattended NIV, which could be responsible for much worse treatment outcomes than observed in controlled studies. Therefore, it seems to be crucial to advocate for NIV usefulness and a minimal acceptable setting at a government or regional level (e.g. "intermediate" dependency units) [21].

It has been proven that implementation of standards can improve treatment of AE-COPD in emergency departments [22]. Most respondents indicated that NIV skills are gained during specialisation training or by themselves from the medical literature, but this was not dependent on region or income level. There 
was also an increasing number of respondents from all economies who gained their skills in NIV provision at an ERS school course and/or during an ERS postgraduate course. This shows that medical employees in all countries are interested in respiratory training and are keen to travel abroad for the highest international standard NIV training.

\section{Study limitations}

One of the most important study limitations is that the survey was not translated from English into other native languages. Thus, we need to take into account that there might have been some misunderstanding about the questions as a result of language barriers. However, the questionnaire was sent to ERS members, which is known to be an English-speaking society. The accuracy and representativeness of our survey can be confirmed by similar epidemiological findings defined by age, profession and setting reported in our previous survey [15] or previous results of the Eurovent survey [2]. Thus, we conclude that language barriers should not have biased our survey results.

Another limitation of our study is the fact that we have no responses from LIEs and only a poor response from Africa (represented by just three countries). We need to take into consideration that, according to the best of our knowledge, until now there has been absolutely no data concerning NIV in Africa and in LIEs. This requires further actions, such as establishing contact with national respiratory societies of those countries or, in cases where no such societies exits, with the relevant ministry of health to reveal whether NIV is not used in those countries or whether the low response was caused by the underrepresentation of LIE members in the ERS. However, considering that the ERS is a global scientific organisation, which incorporates both physicians and allied health professionals from most nations, representing all continents and economies, no responses from LIEs was surprising to some extent. Noting that there are obviously huge differences in the practical implementation of NIV, the survey authors expected that there might be fewer responses from LIEs. Unfortunately, although the invitation to take part in the survey was sent out a number of times, we were not able to achieve a higher response rate.

Another limitation is the overrepresentation of pulmonologists (88\%) and medical doctors (90.7\%). The only way to avoid this would be to create a future survey addressed not only to ERS members, but also to other societies, such as those for anaesthesiologists, intensivists, respiratory nurses and respiratory therapists. The accuracy of our data is supported by the fact that in comparison to other more local surveys, we were able to obtain global data from all continents but unfortunately with the exception of LIEs [10, 12-14]. Moreover, there was a low representation of respiratory nurses and physiotherapist. To some extent, this was foreseen and discussed at the time of survey distribution. It was decided that the survey would be distributed among ERS Respiratory Intensive Care Assembly members and National Delegates for the following reasons. 1) ERS members may be members of more than one assembly and therefore members of the Allied Respiratory Professionals Assembly interested in NIV were probably also members of the Respiratory Intensive Care Assembly. 2) Although most ERS National Delegates are probably not directly involved in NIV provision, they should have a general knowledge about their national situation. Taking into account that in a medical society such as the ERS there is an obvious predominance of medical doctors, we have managed to obtain responses from nurses $(0.7 \%)$, respiratory therapists $(5.3 \%)$ and other healthcare professionals (3.3\%). Moreover, according to our results medical doctors are mostly involved in NIV practical provision (73.3\%). This suggests that respiratory therapists are currently involved only in a minority of countries.

A further study limitation was the fact that we have not asked specific respondents who pointed out the importance of financial issues for solutions and further explanations. This was for two reasons: 1) the study protocol did not aim to change the current situation, which was not known at the study planning stage, and 2) we found that there were larger income- than region-dependent reasons limiting NIV use. Considering the global scale of our survey, it was impossible to address, predict and automatically provide solutions to particular locally important factors limiting NIV use. Obviously, taking into account the different medical systems, the survey results obtained showed huge differentiation among countries. We found that local country-specific constraints may be significantly different from regional issues; therefore, in order to provide solutions, smaller, more detail surveys would have been more useful. Thus, there is an urgent need for an official chronic respiratory failure NIV treatment guideline, similar to the recently published ERS/American Thoracic Society clinical practice guideline for acute respiratory failure [23], but enriched in economic and cost-effectiveness factors.

A final, but unfortunately important study limitation, which was not foreseen at the survey planning stage, was the small number of questions concerning NIV education and training. Although there were no region- or income-related differences, the survey revealed that NIV training took place during a university course for $<20 \%$ of respondents. This was not analysed in detail here because we consider that NIV 
education/training needs another set of questions, which would have resulted in a longer survey and a much lower response rate.

\section{Conclusions}

Financial constraints are still considered as the major factor limiting NIV use in chronic respiratory failure, especially in LMIEs and UMIEs. Although NIV is rarely studied or practically trained at the stage of university education, there are no major (region- or income-related) differences in NIV teaching.

Acknowledgements: We would like to acknowledge all ERS Respiratory Intensive Care Assembly members and ERS National Delegates who completed our questionnaire. Special thanks to the ERS for providing facilities needed to complete this project. We would like to acknowledge Grzegorz Brożek (Dept of Epidemiology, The Medical University of Silesia, Katowice, Poland) for performing statistical analysis.

Conflict of interest: S. Skoczyński has nothing to disclose. R. Scala has nothing to disclose. P. Navalesi reports grants and other funding (royalties) from Intersurgical SpA, grants and personal fees from Maquet, and personal fees from Philips, ResMed and Novartis, outside the submitted work.

\section{References}

1 McKim DA, Road J, Avendano M, et al. Home mechanical ventilation: a Canadian Thoracic Society clinical practice guideline. Can Respir J 2011; 18: 197-215.

2 Lloyd-Owen SJ, Donaldson GC, Ambrosino N, et al. Patterns of home mechanical ventilation use in Europe results from the Eurovent survey. Eur Respir J 2005; 25: 1025-1031.

3 Global Initiative for Chronic Obstructive Lung Disease. Global Strategy for the Diagnosis, Management, and Prevention of Chronic Obstructive Pulmonary Disease. 2017. http://goldcopd.org/gold-2017-global-strategydiagnosis-management-prevention-copd Date last accessed: September 14, 2018.

4 Kaul S, Pearson M, Coutts I, et al. Non-invasive ventilation (NIV) in the clinical management of acute COPD in 233 UK hospitals: results from the RCP/BTS 2003 National COPD Audit. COPD 2009; 6: 171-176.

5 The World Bank. World Bank Country and Lending Groups. 2017. https://datahelpdesk.worldbank.org/ knowledgebase/articles/906519-world-bank-country-and-lending-groups Date last accessed: July 4, 2017.

6 Salvadè I, Domenighetti G, Jolliet P, et al. Perception of non-invasive ventilation in adult Swiss intensive care units. Swiss Med Wkly 2012; 142: w13551.

7 Maheshwari V, Paioli D, Rothaar R, et al. Utilization of noninvasive ventilation in acute care hospitals: a regional survey. Chest 2006; 129: 1226-1233.

8 Simonelli C, Paneroni M, Vitacca M. An implementation protocol for noninvasive ventilation prescription: the physiotherapist's role in an Italian hospital. Respir Care 2013; 58: 662-668.

9 Crimi C, Noto A, Princi P, et al. A European survey of noninvasive ventilation practices. Eur Respir J 2010; 36 : 362-369.

10 Hess DR, Pang JM, Camargo CA Jr. A survey of the use of noninvasive ventilation in academic emergency departments in the United States. Respir Care 2009; 54: 1306-1312.

11 Burns KE, Sinuff T, Adhikari NK, et al. Bilevel noninvasive positive pressure ventilation for acute respiratory failure: survey of Ontario practice. Crit Care Med 2005; 33: 1477-1483.

12 Carlucci A, Richard JC, Wysocki M, et al. Noninvasive versus conventional mechanical ventilation. An epidemiologic survey. Am J Respir Crit Care Med 2001; 163: 874-880.

13 Doherty MJ, Greenstone MA. Survey of non-invasive ventilation (NIPPV) in patients with acute exacerbations of chronic obstructive pulmonary disease (COPD) in the UK. Thorax 1998; 53: 863-866.

14 Sweet DD, Naismith A, Keenan SP, et al. Missed opportunities for noninvasive positive pressure ventilation: a utilization review. J Crit Care 2008; 23: 111-117.

15 Scala R, Windisch W, Köhnlein T, et al. Targeting European Respiratory Society Group activities: a survey of the Noninvasive Ventilatory Support Group. Eur Respir Rev 2014; 23: 258-260.

16 Ornico SR, Lobo SM, Sanches HS, et al. Noninvasive ventilation immediately after extubation improves weaning outcome after acute respiratory failure: a randomized controlled trial. Crit Care 2013; 17: R39.

17 Schnell D, Timsit JF, Darmon M, et al. Noninvasive mechanical ventilation in acute respiratory failure: trends in use and outcomes. Intensive Care Med 2014; 40: 582-591.

18 Stefan MS, Shieh MS, Pekow PS, et al. Epidemiology and outcomes of acute respiratory failure in the United States, 2001 to 2009: a national survey. J Hosp Med 2013; 8: 76-82.

19 Roberts CM, Lopez-Campos JL, Pozo-Rodriguez F, et al. European hospital adherence to GOLD recommendations for chronic obstructive pulmonary disease (COPD) exacerbation admissions. Thorax 2013; 68: 1169-1171.

20 Roberts CM, Stone RA, Buckingham RJ, et al. Acidosis, non-invasive ventilation and mortality in hospitalised COPD exacerbations. Thorax 2011; 66: 43-48.

21 Scala R. Respiratory high-dependency care units for the burden of acute respiratory failure. Eur J Intern Med 2012; 23: $302-308$

22 Sen B, Woollard M, Desira NL. Does the introduction of a COPD pro-forma improve the standards of care delivered by junior doctors in the emergency department. COPD 2010; 7: 199-203.

23 Rochwerg B, Brochard L, Elliott MW, et al. Official ERS/ATS clinical practice guidelines: noninvasive ventilation for acute respiratory failure. Eur Respir J 2017; 50: 1602426. 